Article

\title{
On Nearly Newtonian Potentials and Their Implications to Astrophysics
}

\author{
Abraao J. S. Capistrano ${ }^{1,2}$ (1) \\ 1 Federal University of Latin American Integration, Natural Sciences Interdisciplinary Center, \\ Itaipu Technological Park, P.o.b: 2123, 85867-670 Foz do iguaçu-PR, Brazil; abraao.capistrano@unila.edu.br \\ 2 Casimiro Montenegro Filho Astronomy Center, Itaipu Technological Park, \\ 85867-900 Foz do iguaçu-PR, Brazil
}

Received: 28 February 2018; Accepted: 27 March 2018; Published: 17 April 2018

\begin{abstract}
We review the concept of the slow motion problem in General relativity. We discuss how the understanding of this process may imprint influence on the explanation of astrophysical problems.
\end{abstract}

Keywords: general relativity; gravitational field; astrophysics

\section{Introduction}

Since the publication of the General relativity theory (GRT) in 1915 by Einstein, the problem of motion has been a paramount issue to solve, even with all the triumphs of GRT: the prediction of the deviation of light when it passes through a sufficient matter source like a star, or on the explanation of the advance of perihelion of Mercury, or even the prediction of gravitational waves, recently discovered by direct evidence [1]. The answers firstly came up with a published paper of Einstein, Hoffman and Infeld [2] in 1938, and almost 25 years later, a better discussion on this theme was given with the publication of two important books by Fock [3] and Infeld and Plebanski [4]. In different ways, they showed essentially that the Einstein field equations give rise to the equations of motion. This is quite the contrary to what happens in the Newtonian theory where the equations of field and motion are at first sight well posed uncorrelated concepts. In this paper, we follow this idea and review some foundations of GRT and discuss the process of linearizing Einstein's gravity to Newton's and possible consequences for astrophysical phenomena in a "relativistic slow motion" approach.

\section{The Problem of Motion in GRT}

As is well known, GRT has three fundamental axioms, which are the equivalence principle, the general covariance principle and Einstein's action. The third axiom defines Einstein's field equations where the geodesic equation is derived. The same does not follow from Newton's theory, where the field equation and the equations of motion are two independent principles. It evinces the statement that every classical gravitational theory must have field equations and equations of motion. Thus, one expects to attain the Newtonian limit through GRT, and we have to look for two sets of equations (field and motion).

On the other hand, it was not very clear how the equations of motion were contained in Einstein's equations. Einstein and Grommer [5] discussed that the movement of matter must be through geodesics and the movement of a mass-energy content in a gravitational field must violate field equations. Hence, due to the self interaction of the Einstein gravitational field affecting its own dynamics, the equations of motion appear through geodesics and an independent postulate of motion should not be required. In other words, they represent a necessary condition for the existence of solutions of the field equations. Another interesting point is related to singularities. In Newtonian theory, the singularities come up with the representation of a material point with infinite mass density. 
In GRT, the singularities can be represented by the metric tensor $g_{\mu \nu}=g_{\mu \nu}\left(x^{\alpha}\right)$. Let the metric tensor be an analytic function, and then the quantities related to field can be expanded in a power series endowed with an arbitrary parameter. Infeld and Plebanski [4] proposed the idea that the equation of motion in GRT comes from Einstein's field equations in a different modus faciendi, for which the field equations of GRT were obtained through Newton's equations. Starting from Newton's equations, they took approximations of the metric with the parameter $v / c \ll 1$, and, once the geodesic equations are built, Newton's postulate of motion is not necessary anymore. Accordingly, a specific choice of parameters has to be made for a specific ending. This is the key point that leads to our further analysis.

\subsection{Linearized Gravity and PPN Approximation}

There are several examples in literature of how to linearize GRT reducing it to the Newtonian weak field. The main concept resides in the reduction of a nonlinear strong field to weak field under presuppositions of possible large velocities and pressure, with the condition

$$
g_{\mu \nu}=\eta_{\mu \nu}+\delta h_{\mu \nu}+\mathcal{O}\left(\delta h_{\mu \nu}^{2}\right)
$$

where $\eta_{\mu \nu}$ is the Minkowski flat metric and $h_{\mu \nu}$ is a fraction of the gravitational field with the condition that $h_{\mu v}<<1$. The superior orders of $h_{\mu \nu}^{2}$ are ignored. The dynamics lies on linear terms of $h_{\mu \nu}$. This is the typical "weak field" situation.

As well posed by Infeld and Plebanski [4], it is possible to define other parameters to expand the metric tensor than the one related only to velocity; for instance, the weak field limit that gives the linear gravitational wave equation or the Schwarzschild weak field with the parameter $\frac{1}{r}$. Interestingly, besides all applications nowadays, this concept was used in works of field unification in the 1950s. In 1954, Suraj Gupta [6] proposed a theorem that establishes that every field of spin-2 in the Minkowski space-time can be described by a system of similar structures as Einstein's equations. The process was essentially an application of the linear approximation of the Einstein equations for the gravitational field. Minkowski's flat space-time is regarded as the zero order of Riemannian space approximation. Thus, the physical quantities of GRT are expressed as an infinite series of terms in Minkowski's space-time. Then, Gupta linearized Einstein's equations into Minkowski's space-time with an infinite number of terms in Lagrangian density to obtain a linear theory of gravitation in that space-time. Accordingly, one can compare the mathematical structure of electromagnetism and GRT to understand the difference between their spins. Showing a remarkable resemblance with the linear approximations, the linearized Einstein's equations are written as

$$
\begin{aligned}
& \epsilon^{\alpha \beta} \frac{\partial^{2} g_{\mu v}}{\partial x^{\alpha} \partial x^{\beta}}=\tau_{0} \Theta_{\mu v}, \\
& \Theta_{\mu v ; v}=0,
\end{aligned}
$$

where $\tau_{0}$ is a constant and $\epsilon^{\alpha \beta}$ is a set of quantities given by

$$
\epsilon^{\alpha \beta}=\left(\begin{array}{cccc}
-1 & 0 & 0 & 0 \\
0 & -1 & 0 & 0 \\
0 & 0 & -1 & 0 \\
0 & 0 & 0 & +1
\end{array}\right)
$$

The semicolon symbol denotes a covariant derivative.

The quantity $\Theta_{\mu \nu}$ is a symmetrical energy-momentum pseudotensor, where the metricity condition $g_{\mu v ; v}=0$ applies. Similarly, in Maxwell's theory, we have

$$
\partial^{\nu} \partial_{\nu} A_{\mu}=-(1 / c) j_{\mu},
$$


with the Lorentz gauge

$$
A_{\mu, \mu}=0
$$

where $A_{\mu}$ is the electromagnetic potential and $j_{\mu}$ is the current four-vector. The comma symbol denotes the ordinary derivative. Thus, one can obtain the following set of equations:

$$
\begin{aligned}
& \partial^{\lambda} \partial_{\lambda} U_{\mu v}=\tau_{0} \Omega_{\mu v}, \\
& U_{\mu v ; v}=0, \\
& \Omega_{\mu v ; v}=0,
\end{aligned}
$$

where $U_{\mu \nu}$ is a real symmetrical tensor, $\tau_{0}$ is a coupling constant and $\Omega_{\mu \nu}$ is a conserved symmetrical tensor, which can also be written as $t_{\mu \nu}$. The $t_{\mu \nu}$ tensor represents the tensor for the gravitational field energy-momentum plus the $T_{\mu \nu}$ tensor, which represents the tensor for the energy-momentum of the gauge interactions. The same equation can also be derived from a variational principle, leading to the Lagrangian density

$$
\mathcal{L}=-\frac{1}{2} \frac{\partial U_{\mu v}}{\partial x_{\lambda}} \frac{\partial U_{\mu v}}{\partial x_{\lambda}}+f_{1}+f_{2}+\ldots,
$$

where the infinite terms $\left(f_{1}, f_{2}, \ldots\right)$ compose the energy-momentum $t_{\mu v}$. Accordingly, one can obtain the equations

$$
U_{\mu v}-\frac{1}{2} U u_{\mu v}=\alpha \tau_{\mu v}
$$

where the symbols $u_{\mu v}, U_{\mu v}$, and $U$ can be regarded as a metric-type tensor, a Ricci-type tensor and a scalar-type tensor, respectively. Clearly, this new geometry was a copy of Riemann's geometry with a metric and a associated curvature. The main problem of this process was that Gupta assumed the quadridimensional space-time as a flat space, which led to an incongruence with the two metric tensors $g_{\mu \nu}$ and $u_{\mu \nu}$.

Differently from the linearized gravitation started from a nonlinear field endowed with metric $g_{\mu \nu}$ (simply seen as the first order perturbation of flat Minkowsky space-time), the Parameterized Post Newtonian (PPN) has the presuppositions of the weak field regime and the slow motion condition. It is a successful method to study the reduction of Einstein's gravitational field to a post Newtonian gravity and has been largely applied to study astrophysical phenomena. As GRT is a metric theory, all metric theories are subjected to have a PPN approximation under the conditions on the metric components $g_{44} \sim \mathcal{O}\left(\epsilon^{2}\right), g_{4 i} \sim \mathcal{O}\left(\epsilon^{3 / 2}\right)$ and $g_{i j} \sim \mathcal{O}(\epsilon)$, where $\epsilon$ is a small parameter. The total amount of 10 free parameters from the PPN approximation are defined once the tensor metric can be written in terms of potential functions [7-10].

Despite of all the success of PPN formalism as a traditional mechanism from reducing a larger theory of gravitation (Einstein's gravity) to Newtonian theory, it is based on assumptions that the weak field produced by sources are moving in slow velocities as compared to speed of light. It is important to point out that the PPN expansion is not uniformly valid for large distances [11] and limitations for the study of the dynamics of Pulsars with a complex gravitational regimes (so-called quasi-stationary weak-field, strong-field, highly-dynamical strong-field regime-particulary; it is the case of black holes and neutron stars and the radiation regime as discussed in [12]). Moreover, the PPN finds some important issues:

(A) Near and inside the pulsar (and its companion, if it is also a neutron star), the gravitational field is strong and the weak-field assumption no longer holds. In such cases, the internal gravity is regarded as somewhat frozen.

(B) When it comes to generation of gravitational waves (of wavelength $\lambda_{G W}$ ) and their back-reaction on the orbit (of size $r$ and period $P_{b}$ ), the post-Newtonian approximation is only valid in the near zone $\left(r<<\lambda_{G W}=c P_{b} / 2\right)$, and breaks down in the radiation zone $\left(r>\lambda_{G W}\right)$ where 
gravitational waves propagate and boundary conditions are defined, like the "no incoming radiation" condition [12].

This conceptualization is also motivated, for instance, by the observed correlation between the highest-energy cosmic rays sources and active galactic nuclei [13] and more recently in [14] with the existence of strong gravitational fields in those regions [15-17]. Hence, the observed motion of stars near black holes observed at the nuclei of some of these galaxies indicate that the velocities are small, of the order of a few hundreds of kilometers per second. This requires the description of a slow geodesic motion in the presence of strong gravitational fields. Those facts contribute to reinforce the idea that, in the dark matter problem, GRT cannot be used; for large distances, it fails exactly where the discrepancy occurs and matches the Newtonian results. Hopefully, we may address those problems in reviewing fundamentals of GRT.

\subsection{The Nearly Newtonian Approach}

Interestingly, in the specialised literature, the terms slow motion and weak field have been treated as traditional jargon to get to the Newtonian theory. We explore the possibility of what happens if one works only with the hypothesis of slow motion in an arbitrary gravitational field. Essentially, we follow the same development of the traditional Misner, Thorne and Wheeler's book [18] where we summarize the main aspects in the following, showing that the Newtonian equation of motion appears in the limit of GRT as an option, in which case we also need to impose the weak field condition to Einstein's equations.

Firstly, we assume that the related velocities $v$ are smaller than the speed of light $v \ll c$. As it seems evident for any astrophysical system, a test particle falling into a gravitational field from the exact solution of the Einstein's equations will not have velocity close to the speed of light. For instance, a typical star of the order of the solar mass has peculiar velocity of the order of $250 \mathrm{~km} / \mathrm{s}$, and a test particle around it will try an exterior gravitational field as it gets closer to the star from increments $\delta h_{\mu v}$ of this field in such a way that one can write the metric tensor build up as

$$
\begin{aligned}
& g_{\mu v}=\eta_{\mu v}+\delta h_{\mu v}+\mathcal{O}\left(\delta h_{\mu \nu}^{2}\right), \\
& g^{\mu v}=\eta^{\mu v}-\delta h^{\mu v}+\mathcal{O}\left(\delta h_{\mu v}^{2}\right) .
\end{aligned}
$$

On the other hand, the slow motion condition should not be bounded to the gravitational field and also not bounded immediately to the Newtonian limit. In the following, we summarize briefly the reduction of GRT to Newtonian limit from the deviation equation obtaining the very known Poisson equation obtaining the postulate of field. Considering a fraction of time $\delta t$ in which a test particle travels a distance $\delta x^{i}$, where $(i=1,2,3)$ in low speed in such a way,

$$
\begin{gathered}
\delta x^{i} \sim v \delta t \Longrightarrow \delta x^{i} \approx \lambda \delta x^{4}, \\
\frac{\lambda}{\delta x^{i}} \sim \frac{1}{\delta x^{4}},
\end{gathered}
$$

where $\lambda=\frac{v}{c}$ and $\delta x^{4}=c \delta t$.

Defining an arbitrary function $f$,

$$
\frac{\partial f}{\partial x^{4}} \sim \lambda \times \frac{\partial f}{\partial x^{i}} .
$$

In addition, if we consider two test particles in free fall, one particle is at a point $x^{i}+\xi^{i}$ and the other at $x^{i}$. Accordingly, using the relative acceleration between them, one obtains

$$
\frac{d^{2} \xi^{i}}{d t^{2}}=\frac{d^{2}\left(x^{i}+\xi^{i}\right)}{d t^{2}}-\frac{d^{2} x^{i}}{d t^{2}}=-\left.\frac{\partial \Phi}{\partial x^{i}}\right|_{x^{i}+\xi^{i}}+\left.\frac{\partial \Phi}{\partial x^{i}}\right|_{x^{i}}=-\frac{\partial^{2} \Phi}{\partial x^{i} \partial x^{j}} \xi^{j}
$$


noting that $\frac{\partial^{2} \Phi}{\partial x^{i} \partial x^{j}}$ is the Laplacian of the gravitational potential. Accordingly, one can write the Christoffel symbols

$$
\Gamma_{\mu \nu}^{\lambda}=\frac{1}{2} \eta^{\lambda \sigma}\left(\delta h_{\mu \sigma, v}+\delta h_{v \sigma, \mu}-\delta h_{\mu v, \sigma}\right)+\mathcal{O}\left(\delta h_{\mu \nu}^{2}\right) .
$$

Moreover, considering the deviation equation, we have

$$
\frac{D^{2} \xi^{i}}{d \tau^{2}}=\frac{d^{2} \xi^{i}}{d t^{2}}=-\mathcal{R}_{4 j 4}^{i} \xi^{i}
$$

and the Ricci tensor in a form

$$
\mathcal{R}_{i j}=\Gamma_{i k, j}^{k}-\Gamma_{i j, k}^{k}+\mathcal{O}\left(\delta h_{i j}^{2}\right)
$$

such that

$$
\mathcal{R}_{44}=\mathcal{R}_{4 i 4}^{i}=-\frac{1}{2} \delta h_{44, i i}+\mathcal{O}\left(\delta h_{i j}^{2}\right)=-\nabla^{2} \Phi+\mathcal{O}\left(\delta h_{i j}^{2}\right),
$$

and, with the Einstein equation with material source

$$
\mathcal{R}_{\mu v}-\frac{1}{2} \mathcal{R} g_{\mu v}=8 \pi T_{\mu v},
$$

where $T_{\mu v}$ is the energy-momentum tensor. Hence, one can find $\mathcal{R}_{44}=4 \pi \rho$ that leads to Poisson equation

$$
\nabla^{2} \Phi=4 \pi \rho
$$

where $\rho$ denotes the matter density.

This equation is fundamental for completing the reduction of GRT to the Newtonian limit once the reconstruction of the postulate of motion by the geodesic equation must be done. It is important to note that the Newton's reduction to GRT is an option and depends on the retrieval of the basic postulates of field and motion. However, what would happen if only the geodesic equation is regarded for the dynamics, leaving intact the Einstein equations and geodesic deviation? The resulting potential related to this type of approach is called "nearly-Newtonian" potential [18]. The denomination "nearly-Newtonian" refers to the fact that the limit is not complete. If one looks at the geodesic equation alone, one may find interesting quantitative and qualitative results.

In order to check this assumption, let us consider again a particle that moves in slow motion in a timelike geodesics such that $x^{\mu}=x^{\mu}(\tau)$ in which its own gravitational field is weak enough with respect to an external gravitational source. Hence, we write

$$
\frac{d^{2} x^{\mu}}{d \tau^{2}}+\Gamma_{\lambda v}^{\mu} \frac{d x^{\lambda}}{d \tau} \frac{d x^{v}}{d \tau}=0
$$

and, parameterizing in terms of the coordinate $x^{4}=-c t$, it is straightforward to show that

$$
\frac{d^{2} x^{i}}{c^{2} d t^{2}} \simeq-\Gamma_{44}^{i}
$$

where

$$
\Gamma_{44}^{i}=-\frac{1}{2}\left(2 \frac{\partial \delta h_{4 i}}{\partial x^{4}}-\frac{\partial \delta h_{44}}{\partial x^{i}}\right),
$$

which is a static field such that $\frac{\partial \delta h_{4 i}}{\partial x^{4}}=0$, thus

$$
\Gamma_{44}^{i} \simeq \frac{1}{2} \frac{\partial \delta h_{44}}{\partial x^{i}} .
$$

Hence,

$$
\frac{d^{2} x^{i}}{d t^{2}} \simeq \frac{1}{2} c^{2} \frac{\partial \delta h_{44}}{\partial x^{i}}
$$


As the geodesic equations come from Einstein's equations, one can postulate a scalar potential defined by

$$
\frac{d^{2} x^{i}}{d t^{2}} \stackrel{d e f}{=}-\frac{\partial \Phi_{q N}}{\partial x^{i}},
$$

where $\Phi_{q N}$ denotes the nearly Newtonian scalar potential as felt by a test particle. In addition, considering a free falling test particle in slow motion, the gravitational field acts independently from the motion of the particle in an infinite increments such that the metric tensor should be given by

$$
g_{\mu v} \approx \eta_{\mu v}+\delta h_{\mu v}+\left(\delta h_{\mu v}\right)^{2}+\cdots
$$

in which it allows a different field than the Newtonian one. Accordingly, as the strength of the field grows, these increments (perturbations) of the metric can be summed from $\delta h_{44}=0$ to a finite value $\delta h_{\mu v}$ to be integrated to give the formula

$$
\Phi_{q N}=-\frac{1}{2} c^{2} \int_{0}^{\delta h_{44}} d\left(\delta h_{44}^{\prime}\right)=-\frac{1}{2} c^{2} \delta h_{44} .
$$

Accordingly,

$$
\Phi_{q N}=-\frac{1}{2} c^{2}\left(1+g_{44}\right) .
$$

It is important to stress that both Equations (13) and (16) are obtained from two independent paths. In other words, we can work with Equation (16) independently and the Gaussian theorem cannot be applied to the potential $\Phi_{q N}$ once Equation (13) is not restored. This kind of situation only reflects partially that "passive" aspects of gravity (response of matter to gravity) can be derived [18]. For instance, we can apply Equation (16) to find the geodesic motion of a free falling star under the gravitational field in the galactic plane, since the slow motion of the geodesic equation is not invariant under diffeomorphisms. In this case, if assuming that the gravitational field of the galaxy should be weak, like that of a point source, the predominant gravitational field is given by the exterior Schwarzschild solution, as seen from a large distance from the galaxy nucleus. In spherical coordinates, we can write the metric component $g_{44}=-(1-2 M / r)$, and find the related potential

$$
\varphi\rfloor_{\text {Newton }}=-M / r, \quad r>>r_{0},
$$

which is equivalent to the Newtonian gravitational potential produced by a distant mass $M$, determined by the Newtonian weak field.

We point out that this scalar potential $\Phi_{q N}$ represents an intermediate field since the summation of the perturbative terms to the metric occurs only in component $g_{44}$ of the metric, which still carries nonlinearity effects. It should be told that Einstein's equations and the geodesic equations are intact. It is important to remember that the recovery of Newtonian postulates of field and motion are independent. Therefore, the scalar potential generated from the field and motion equations are also independents. When we restore the field equation (postulate of field), as shown by the procedure to obtain Equation (13), we make the theory linear, breaking the nonlinearity of the Einstein equations. On the other hand, when we restore only the equation of motion (postulate of motion), we have only the generalized covariance, but nonlinearity is maintained in such a way through the component $g_{44}$, once any dynamical systems propagate their nonlinear effects exerting qualitative effects on their solutions. This comes from the fact that the affine connection has different degrees in the main equations of GRT. The geodesic equation has a linear affine connection, which produces a smoother gravitational field than the other equations. On the other hand, in terms of Einstein's equations, the connection is on the fourth power in this particular set of equations and the deviation equation is quadratic in connection. The symmetry group of Equation (16) is similar to the generalized Galilean group, where the Newtonian potential is replaced by 1 . Since the system is no longer diffeomorphic invariant, the metric should be written in coordinates that are consistent with the metric symmetry 
of the local gravitational field in question. Thus, Equation (16) by construction does not need a non-symmetric metric, once the metric tensor can be thought as a combination of a symmetric and an antisymmetric component, i.e, $g_{\mu \nu}=\frac{1}{2}\left(g_{[\mu \nu]}+g_{(\mu \nu)}\right)$, which considered Einstein himself [19] in a search of a unified theory of physics.

Einstein's original idea was that the antisymmetric part was regarded as the electromagnetic field denoted by a antisymmetric rank-2 tensor. In theoretical physics, an interesting approach was proposed by J. W. Moffat, the so-called the nonsymmetric gravitational theory (NGT), which is a classical theory of gravitation in which the antisymmetric part does not necessarily represent the electromagnetic field, and may be generalized to a fundamental new field [20], later updated to a proposal that the skew symmetric part is a massive Proca field equation [21] used as a basis for a solution of the dark matter problem. Until now, such a unified field was not found as well as a proper solution of the dark matter problem, but research on non-symmetric metrics are still in progress with a focus on several problems, for instance, the dark matter problem itself [22], massive gravity [23], thin-shell wormholes [24], connections with skew-symmetric torsion on non-symmetric Riemannian manifold [25] and Pioneer anomalous acceleration [26].

\section{Astrophysical Applications}

Instead of making an application of approximation methods of GRT, the nearly Newtonian approximation may provide a simpler option to study astrophysical phenomena. In recent years, we have been focused on the apsidal precession (perihelion) problem applied for several celestial bodies including planets, comets and asteroids with satisfactory results $[27,28]$.

Interestingly, axial symmetry has revealed itself to be quite interesting to several applications and is an important tool to describe the perihelion problem mathematically. Weyl showed that the cylinder solution is diffeomorphic to a Schwarzschild's solution and the metric does not lose its asymptotes, as shown in [29-31], and is also asymptotically flat [29-33]. Particularly, the Weyl metric describes a cylinder given by the line element

$$
d s^{2}=e^{\lambda-\sigma} d r^{2}+r^{2} e^{-2 \sigma} d \phi^{2}+e^{2(\lambda-\sigma)} d z^{2}-e^{2 \sigma} d t^{2}
$$

where $\lambda=\lambda(r, z)$ and $\sigma=\sigma(r, z)$. The exterior gravitational field in the cylinder outskirts is given by Einstein's vacuum equations

$$
\begin{aligned}
& -\lambda_{, r}+r \sigma_{, r}^{2}-r \sigma_{, z}^{2}=0, \\
& -\sigma_{, r}-r \sigma_{, r r}-r \sigma_{, z z}=0, \\
& \lambda_{, r r}+\lambda_{, z z}+\sigma_{, r}^{2}+\sigma_{, z}^{2}=0, \\
& 2 r \sigma_{, r} \sigma_{, z}=\lambda_{, z} .
\end{aligned}
$$

To solve the nonlinear system [30], we impose the condition that its height $h_{0}$ is smaller than its radius $R_{0}$, i.e., $h_{0}<<R_{0}$ to reduce the cylinder to its circular basis to describe the orbit of a test particle towards the sun. Other types of approximation are currently being studied [34] as well as the dynamics of relativistic disks [35-39]. As a result, one can obtain the formula for the perihelion advance as

$$
\delta \phi=\delta \phi_{s c h} \pm \delta \epsilon^{4} \sqrt{\frac{G M\left(1-\epsilon^{2}\right)}{a^{3}}}
$$

where we denote $\delta \phi_{s c h}=\frac{6 \pi G M}{c^{2} a\left(1-\epsilon^{2}\right)}$, $a$ is the semi-major axis and $\epsilon$ denotes the eccentricity of the orbits. The $\delta$ denotes a unitary length parameter to maintain the correct dimensionality of Equation (22). This result allows for obtaining two solutions for the angle deviation $\delta \phi$ that depends on the sign that indicates the maximum/minimum values for the $\delta \phi$ variation. If the eccentricity of the orbit is null or too small, the equation is reduced to the standard Schwarzschild one. Table 1 shows a comparison with the observed precession, PPN and the nearly Newtonian approach for select celestial bodies. 
Accordingly, one has $\delta \phi^{(+)}$and $\delta \phi^{(-)}$for plus and minus sign, respectively, which allow for studying retrograde precessions eventually. This theme was explored in [28] in the description of the anomalous apsidal precession of planets. This subtle effect of very small order ( -6 miliarcsec) was indicated in $[40,41]$ using the EPM2008 ephemerides, suggesting that a retrograde precession of Saturn may have a nonzero statistical significance. Using the nearly Newtonian solution, the obtained results were compared to different observations on Ephemerides of the Planets and the Moon (EPM2008) [40], (EMP2011) [42,43] and on Planetary and Lunar Ephemeris (INPOP10a) [44].

Table 1. Comparison between the values for secular precession of Mercury in units of arcsec/century of the standard (Einstein) perihelion precession $\delta \phi_{\text {sch }}$ [45] and the nearly Newtonian solution $\delta \phi_{\text {model }}$. The $\delta \phi_{o b s}$ stands for the secular observed perihelion precession in units of arcsec/century adapted from [46] by adding a supplementary precession corrections from EPM2011 [42,43]—in addition, two Jupiter-family comets 26P/Grigg-Skjellerup and 22p/Kopff [47-49].

\begin{tabular}{ccccc}
\hline Object & $\delta \boldsymbol{\phi}_{\text {obs }}\left({ }^{(\prime} . c y^{-\mathbf{1}}\right)$ & $\delta \boldsymbol{\phi}_{\text {sch }}\left({ }^{\prime \prime} . c y^{-\mathbf{1}}\right)$ & \multicolumn{2}{c}{$\delta \boldsymbol{\phi}_{\text {model }}\left({ }^{\prime \prime} . c y^{-\mathbf{1}}\right)$} \\
\hline & & & $\delta \boldsymbol{\phi}^{+}$ & $\delta \boldsymbol{\phi}^{-}$ \\
\hline Mercury & $43.098 \pm 0.503$ & 42.97817 & 43.1047 & 42.8569 \\
26P/Grigg-Skjellerup & 0.54 & 0.4106 & 0.430490 & 0.391913 \\
22p/Kopff & 0.288 & 0.2474 & 0.255405 & 0.240378 \\
\hline
\end{tabular}

In Table 2, a comparison is shown between the values for anomalous apsidal precession $\delta \phi_{a n o m}^{a, b, c}$ of selected planets in units of miliarcsec/century to the EPM2088 and EPM2011 [42,43] and the INPOP10a planetary ephemerides [44], respectively.

Table 2. Comparison between the values for anomalous apsidal precession for selected planets.

\begin{tabular}{ccccccc}
\hline Object & $\delta \boldsymbol{\phi}_{\text {anom }}^{a}$ & EPM2008 & $\delta \boldsymbol{\phi}_{\text {anom }}^{b}$ & EPM2011 & $\delta \boldsymbol{\phi}_{\text {anom }}^{c}$ & INPOP10a \\
\hline Mercury & -3.7945 & $-3.6 \pm 5.0$ & -2.0237 & $-2.0 \pm 3.0$ & 0.4047 & $0.4 \pm 0.6$ \\
Earth & -0.1869 & $-0.2 \pm 0.4$ & 1.8694 & $0.19 \pm 0.19$ & -0.1669 & $-0.2 \pm 0.9$ \\
Saturn & -5.7876 & $-6.0 \pm 2.0$ & -0.2829 & $-0.32 \pm 0.47$ & 0.1910 & $0.15 \pm 0.68$ \\
\hline
\end{tabular}

Another application was to use the same symmetry to deal with the rotation curve problem on low brightness galaxies (LSBGs) and dwarf galaxies [50]. The rotation curves problem basically consists of the discrepancy observed velocities in the faint outskirts of galaxies and those expected from Newtonian predictions [51]. The justification of this problem is commonly associated with the dark matter (see references for review in [52,53]), which refers to a non-interacting large amount of non-baryonic mass but gravitationally important in such a large amount that it would form a huge spherical halo around galaxies.

Without going into the debate over whether dark matter exists or not, we focused our attention on the visible baryonic distribution obtaining satisfactory results. Interestingly, the baryonic-only models have been favoured by recent observations on rotation curves [54], where they state that mass models of luminous matter heavily affect the rotation curve shapes. Moreover, we found that an effective velocity is given by

$$
v_{e f f}(r)=\sqrt{\frac{G M(r)}{r}+\frac{G M(r)}{2 R_{0}} \beta_{0}\left(\frac{r}{R_{0}}\right)^{k_{0}}},
$$

where the mass function $M(r)$ represents the visible mass profile that decays exponentially within a distance $r$ from the center of the galaxy to the star, and is given by

$$
M(r)=M_{\star}\left[1-\left(1+\frac{r}{r_{c}}\right) \exp \left(-\frac{r}{r_{c}}\right)\right],
$$


where $r_{c}$ is the scale length parameter and $M_{\star}=1.4 M_{H I}+M_{\text {disk }}$ [55], and $M_{H I}$ is the mass of the hydrogenous $21 \mathrm{~cm}$ lines that go through the galactic disk up to the outer disk.

In Figure 1 it is shown examples of interesting fitting curves for the galaxies LSB ESO01400040 and the dwarf UGC 6900 with observational data points extracted from [56,57], respectively.
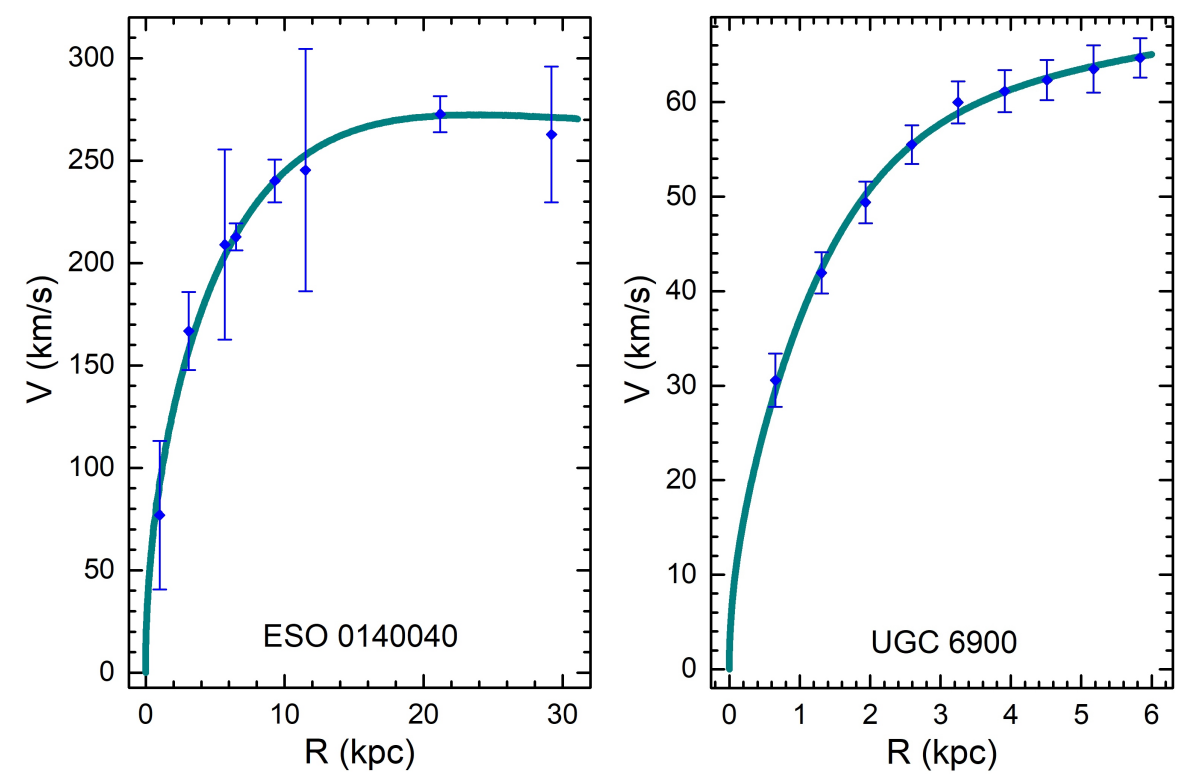

Figure 1. Rotation curves of two selected galaxies. The analysis on goodness-of-fitting to data leads to reduced $\chi_{\text {red }}^{2}$ of $0.025 \pm 0.218$ and $0.297 \pm 0.0780$, respectively [50].

Specifically in the Weyl cylindrical symmetry, it is interesting to notice that, regardless of velocity arguments, the diffeomorphism invariance of GRT also violates the condition that the cylinder height $h_{0}$ is much smaller than its radius $R_{0}$; then, it can no longer be transformed into a Schwarzschild solution. This is an example of the equivalence problem in GRT on how to know if two solutions of Einstein's equations do not describe the same gravitational field written in different coordinates. The application of Cartan's equivalence to GRT solves this issue by analysing the Riemann tensor and their covariant derivatives up to the seventh order, which must be equal. This characteristic of the method turns out to be an interesting new arena for studying more astrophysical applications once the astrophysical objects have different forms and the metric tensor is not restricted to be symmetric, e.g., non-symmetric stars, since they are deformed by their rotation and magnetic field, and eventually do collapse to non-symmetric black holes. Actually, in the realm of GRT, the key point is to obtain the Weyl cylindric potentials to get a Newtonian-like potential to study possible gravitational effects related to a specific problem. This Newtonian analogue of Weyl metric potential has been extensively studied in literature and can be found, e.g, in gravitation textbooks such as in [33,58,59], once the so-called Weyl metrics define a class of static and axisymmetric solutions of Einstein's equations.

It is important point to out that this differs from the paper in Reference [60], since GRT is then regarded not to provide a solution for the rotation curve problem. In our approach, differently from the referred paper, we use an approximate stationary axially symmetric solutions of the $4 \mathrm{D}$ vacuum Einstein equations and study the kinematical behaviour of a test particle calculating the resulting potential produced by the geodesic equation alone. Weyl's seminal paper showed that the cylinder solution is diffeomorphic to a Schwarzschild's solution. In this process, the metric does not lose its asymptotes; in other words, the metric is asymptotically flat [29-32]. The exterior gravitational field in the cylinder outskirts is given by Einstein's vacuum equations and the diffeomorphism invariance of GRT is broken down by the condition that the cylinder height $\mathrm{H}$ is much smaller than its radius $\mathrm{R}$ being reduced to its circular basis, which can be smoothly deformed by the expansion of the metric 
parameters so that the solution must be written in cylindrical coordinates. To analyse effects of the lack of the diffeomorphism invariance, we start with solving the nonlinear system given generated by vacuum Einstein equations. It is to important to point out that we do not use a thin disk model in order to avoid the discontinuity on the $z=0$ plane. A correct relativistic thin disk model was extensively studied in [35-39] for a mass distribution with Weyl's exact solutions of Einstein equations. On the other hand, in our paper, we study approximate solutions of this metric by expanding its coefficient functions (or potentials), which essentially refers to the orbit of a test particle and is not related to the distribution of matter in galaxy itself, thus any problems with asymptotes around $z=0$ cease to be. Naturally, a mass model must be applied. Thus, an effective galaxy model is constructed by the Blumenthal mass model [55], which allows that the passage from the point-like source to an extended one is correct in a linearized framework with a Newtonian mass M(r).

\section{Conclusions}

In this paper, we review the possibility of studying astrophysical phenomena taking into account the slow motion condition with arbitrary gravitational field, i.e, not necessarily being as weak as Newtonian gravity. Regarding this matter, we have discussed the standard approximations of GRT to Newtonian theory, as the linear approximation that the expansion of the metric tensor is limited to the first order, and the PPN approximation in which the tensor metric must be symmetric and must be perturbed by a small fraction (weak field condition) under slow motion condition. Needless to say, the PPN approximation has been a successful mechanism to get rid of gravitational phenomena on the Solar system scale. On the other hand, we have focused our attention on the process of reducing GRT to Newtonian theory. The Newtonian theory is recovered from GRT under the condition that one can explicitly recover two main postulates of the theory: the postulate of field and the postulate of motion. They are separated and independent principles in Newtonian theory. In GRT, these concepts are a medley in Einstein equations. This kind of situation was not clear since the publication of the Einstein's GRT seminal paper and the answers came up with the works of [3,4]. On the other hand, if one chooses not to recover Newtonian theory entirely per se and only focus on the resulting potential obtained from the geodesic equations under slow motion conditions restating the postulate of motion, we attain, using Wheeler's words [18], a nearly-Newtonian potential, which means that this field lies somewhere between GRT and Newton's gravity and does not have a spherical symmetry (we do not have a defined symmetry a priori) and the Gaussian theorem cannot be applied.

We relate the existence of such potential to the fact that, in GRT, the equations of motion are a consequence of the nonlinearity of Einstein's equations, making a contrast with Newtonian gravity. In particular, when the nearly Newtonian potential is derived from a vacuum solution of Einstein's equations, the slow motion of a test particle or a falling star is affected by the interaction of its own gravitational field. From the impositions made on the geodesic equations, one can obtain Equation (16) that relates an arbitrary potential $\Phi$ with the component $g_{44}$ of the metric that carries part of the nonlinearity of Einstein's equations, which means that making the choice of an adequate geometry becomes a very important matter, since the diffeomorphism transformations are not allowed. This is a remarkable feature of the model that allowed us to apply it to different astrophysical applications, e.g., on the dark matter problem and the perihelion precession of planets, asteroids and comets, obtaining promising results. These results motivate proceeding with further testing of the model to explore new prospects for relativistic astrophysics.

Acknowledgments: We thank the anonymous referees for their criticisms and suggestions that improved this paper in its final form.

Conflicts of Interest: The author declares no conflict of interest. 


\section{Abbreviations}

The following abbreviations are used in this manuscript:

GRT General Relativity theory

PPN Post-Newtonian approximation

LSB Low Surface brightness (galaxies)

\section{References}

1. Abbott, B.P.; Abbott, R.; Abbott, T.D.; Abernathy, M.R.; Acernese, F.; Ackley, K.; Adams, C.; Adams, T.; Addesso, P.; Adhikari, R.X.; Adya, V.B.; et al. Properties of the binary black hole merger GW150914. Phys. Rev. Lett. 2016, 116, 241102, doi:10.1103/PhysRevLett.116.241102. [CrossRef]

2. Einstein, A.; Infeld, L.; Horffmann, B. The gravitational equations and the problem of Motion. Ann. Math. 1938, 39, 65-100, doi:10.2307/1968714. [CrossRef]

3. Fock, V. The law of gravitation and the laws of motion. In The Theory of Space, Time and Gravitation; Pergamon Press Ltd.: New York, NY, USA, 1964; pp. 238-317; ISBN 9781483184906.

4. Infeld, L.; Plebanski, J. Motion and Relativity; Pergamon Press Ltd.: New York, NY, USA, 1960; ISBN 9781483280387.

5. Einstein, A.; Grommer, J. Allgemein Relativitätstheorie und Bewegungsgesetz. Sitzber. Preuss. Akad. Wiss. Phys. Math. 1927, 2, 1-11.

6. Gupta, S.N. Eletromagnetism and gravitation. Phys. Rev. 1954, 96, 1683, doi:10.1103/PhysRev.96.1683. [CrossRef]

7. Will, C.M . Theory and Experiment in Gravitational Physics; Cambrigde University Press: Cambrigde, UK, 1993; p. 396; ISBN 0521439736.

8. Will, C.M . The Confrontation between General Relativity and Experiment. Living Rev. Relativ. 2006, 9, 3-13, doi:10.12942/lrr-2014-4. [CrossRef]

9. Smalley, L.L. Post-Newtonian approximation of the Poincaré gauge theory of gravitation. Phys. Rev. D 1980, 21, 328, doi:10.1103/PhysRevD.21.328. [CrossRef]

10. Hohmann, M. Parameterized post-Newtonian limit of Horndeski's gravity theory. Phys. Rev. D 2015, 96, 064019, doi:10.1103/PhysRevD.92.064019. [CrossRef]

11. Burke, W.L. The Coupling of Gravitational Radiation to Nonrelativistic Sources. Ph.D. Thesis, California Institute of Technology, Pasadena, CA, USA, 1969.

12. Wex, N. Testing the Motion of Strongly Self-Gravitating Bodies with Radio Pulsars. In Fundamental Theories of Physics; Puetzfeld, D., Lämmerzahl, C., Schutz, B., Eds.; Springer International Publishing: New York, NY, USA, 2015; p. 653.

13. The Pierre Auger Collaboration. Correlation of the highest-energy cosmic rays with nearby extragalactic objects. Science 2007, 318, 938-943, doi:10.1126/science.1151124. [CrossRef]

14. The Pierre Auger Collaboration; Aab, A.; Abreu, P.; Aglietta, M.; Al Samarai, I.; Albuquerque, I.F.M.; Allekotte, I.; Almela, A.; Alvarez Castillo, J.; Alvarez-Muñiz, G.A.; et al. Observation of a large-scale anisotropy in the arrival directions of cosmic rays above $8 \times 10^{18} \mathrm{eV}$. Science 2017, 357, 1266-1270, doi:10.1126/science.aan4338. [CrossRef]

15. Richstone, D.; Ajhar, E.A.; Bender, R.; Bower, G.; Dressler, A.; Faber, S.M.; Filippenko, A.V.; Gebhardt, K.; Green, R.; Ho, L.C.; et al. Supermassive Black Holes and the Evolution of Galaxies. Nature 1998, 395, A14-A19.

16. Ferrarese, L.; Merrit, D. Supermassive black-holes. Phys. World 2002, 15, 41, doi:10.1088/2058-7058/15/6/43. [CrossRef]

17. Ferrarese, L.; Ford, H. Supermassive Black Holes in Galactic Nuclei: Past, Present and Future Research. Space Sci. Rev 2005, 116, 523-627, doi:10.1007/s11214-005-3947-6. [CrossRef]

18. Misner, C.; Thorne, K.S.; Wheeler, J.A. The Newtonian limit. In Gravitation; W. H. Freeman and Company: New York, NY, USA, 1973; pp. 412-416; ISBN 0-7167-0344-0.

19. Einstein, A. The Meaning of Relativity; Princeton University Press: Princeton, NJ, USA, 1953; ISBN 9780691164083.

20. Moffat, J.W. New theory of gravitaion. Phys. Rev. D 1979, 19, 3554-3558, doi:10.1103/PhysRevD.19.3554. [CrossRef] 
21. Moffat, J.W. Non symmetrical gravitational theory. Phys. Lett. B 1995, 355, 447-452, doi:10.1016/03702693(95)00670-G. [CrossRef]

22. Van Meter, J.R. Dark-matter-like solutions to Einstein's unified field equations. Phys. Rev. D 2018, 97, 044018, doi:10.1103/PhysRevD.97.044018. [CrossRef]

23. Dalmazi, D.; Fortes, H.G.M. Nonsymmetric tensor description of massive spin-2 particles in a curved background. Phys. Rev. D 2017, 95, 065028, doi:10.1103/PhysRevD.95.065028. [CrossRef]

24. Svítek, O.; Tahamtan, T. Nonsymmetric dynamical thin-shell wormhole in Robinson-Trautman class. Eur. Phys. J. C 2018, 78, 167, doi:10.1140/epjc/s10052-018-5628-0. [CrossRef]

25. Ivanov, S.; Zlatanović, M. Connections on non-symmetric (generalized) Riemannian manifold and gravity. Class. Quantum Gravity 2015, 33, 075016, doi:10.1088/0264-9381/33/7/075016. [CrossRef]

26. Kalinowski, M.W. Pioneer 10 and 11 Spacecraft Anomalous Acceleration in the light of the Nonsymmetric Kaluza-Klein (Jordan-Thiry) Theory. Fortschr. Phys. 2015, 63, 11-12, doi:10.1002/prop.201500046. [CrossRef]

27. Capistrano, A.J.S.; Roque, W.L. ; Valada, R.S. Weyl conformastatic perihelion advance. Mon. Not. R. Astron. Soc. 2014, 444, 1639-1646, doi:10.1093/mnras/stu1567. [CrossRef]

28. Capistrano, A.J.S.; Penãgos, J.A.M. ; Alárcon, M.S. Anomalous precession of planets for a Weyl conformastatic solution. Mon. Not. R. Astron. Soc. 2016, 463, 1587-1591, doi:10.1093/mnras/stw1958. [CrossRef]

29. Weyl, H. Zur Gravitationstheorie. Ann. Phys. 1917, 54, 117-145, doi:10.1002/andp.19173591804. [CrossRef]

30. Rosen, N. A Particle at Rest in a Static Gravitational Field. Rev. Mod. Phys. 1949, 21, 503, doi:10.1103/RevModPhys.21.503. [CrossRef]

31. Zipoy, M.D. Topology of some spheroidal metrics. J. Math. Phys. 1966, 7, 1137-1143, doi:10.1063/1.1705005. [CrossRef]

32. Gautreau, R.; Hoffman, R.B.; Armenti, A. Static multiparticle systems in general relativity. Il Nuovo Cimento B 1972, 7, 71, doi:10.1007/BF02827038. [CrossRef]

33. Hoenselaers, C.; Kramer, D.; Stephani, H.; MacCallum, M. Exact Solutions of Einstein's Field Equations; Cambridge University Press: Cambridge, UK, 2003; ISBN-13 978-0521467025.

34. Vieira, R.S.S.; Ramos-Caro, J.; Saa, A. Vertical stability of circular orbits in relativistic razor-thin disks. Phys. Rev. D 2016, 94, 104016, doi:10.1103/PhysRevD.94.104016. [CrossRef]

35. González, G.A.; Gutiérrez-Piñeres, A.C.; Ospina, P.A. Conformastatic disk-haloes in Einstein-Maxwell gravity. Phys. Rev. D 2008, 78, 064058, doi:10.1103/PhysRevD.78.064058. [CrossRef]

36. Gutiérrez-Piñeres, A.C.; González G.A.; Quevedo, H. Finite axisymmetric charged dust disks in conformastatic spacetimes. PRD 2008, 87, 044010, doi:10.1103/PhysRevD.87.044010. [CrossRef]

37. Ujevic, M.; Letelier, P.S. Stability of general relativistic geometric thin disks. Phys. Rev. D 2004, 70, 084015, doi:10.1103/PhysRevD.70.084015. [CrossRef]

38. Ujevic, M.; Letelier, P.S. Stability of general relativistic static thick disks: the isotropic Schwarzschild thick disk. Gen. Relativ. Grav. 2001, 39, 1345-1365, doi:10.1007/s10714-007-0438-y. [CrossRef]

39. Vogt, D.; Letelier, P.S. Exact general relativistic discs and the advance of perihelion. Mon. Not. R. Astron. Soc. 2008, 384, 834-842, doi:10.1111/j.1365-2966.2007.12771.x. [CrossRef]

40. Pitjeva, E.V. Ephemerides EPM2008: The Updated Models, Constants, Data. In Proceedings of the Journées "Systémes de Référence Spatio-Temporels" and X Lohrmann-Kolloquium, Dresden, Germany, 22-24 September 2008. Available online: http://syrte.obspm.fr/jsr/journees2008/pdf/ (accessed on 20 May 2016).

41. Iorio, L. The recently determined anomalous perihelion precession of Saturn. Astron. J. 2009, 137, 3615, doi:10.1088/0004-6256/137/3/3615. [CrossRef]

42. Pitjeva, E.V.; Pitjev, N.P. Relativistic effects and dark matter in the Solar system from observations of planets and spacecraft. Mon. Not. R. Astron. Soc. 2013, 432, 3431-3437, doi:10.1093/mnras/stt695. [CrossRef]

43. Pitjev, N.P.; Pitjeva, E.V. Constraints on dark matter in the solar system. Astron. Lett. 2013, 39, 141-149, doi:10.1134/S1063773713020060. [CrossRef]

44. Fienga, A.; Laskar, J.; Kuchynka, P.; Manche, H.; Desvignes, G.; Gastineau, M.; Cognard, I.; Theureau, G. The INPOP10a planetary ephemeris and its applications in fundamental physics. Celest. Mech. Dyn. Astron. 2011, 111, 363-385, doi:10.1007/s10569-011-9377-8. [CrossRef]

45. Wilhelm, K.; Dwivedi, B.N. Secular perihelion advances of the inner planets and asteroid Icarus. New Astron. 2014, 31, 51-55, doi:10.1016/j.newast.2014.02.007. [CrossRef] 
46. Nambuya, G.G. Azimuthally symmetric theory of gravitation-I. On the perihelion precession of planetary orbits. Mon. Not. R. Astron. Soc. 2010, 403, 1381-1391, doi:10.1111/j.1365-2966.2009.16196.x. [CrossRef]

47. Busemann, H.; Nguyen, A.N.; Cody, G.D.; Hoppe, P.; David, A.L.D.; Rhond, K.; Stroud, M.R.; Zega, T.J.; Nittler, L.R. Ultra-primitive interplanetary dust particles from the comet 26P/Grigg-Skjellerup dust stream collection. Earth Planet. Sci. Lett. 2009, 288, 44-57, doi:10.1016/j.epsl.2009.09.007. [CrossRef]

48. Levison, H.F.; Terrell, D.; Wiegert, P.A.; Dones, L.; Duncan, M.J. On the origin of the unusual orbit of Comet 2P/Encke. Icarus 2006, 182, 161-168, doi:10.1016/j.icarus.2005.12.016. [CrossRef]

49. Moreno, F.; Pozuelos, F.; Aceituno, F.; Casanova, V.; Sota, A.; Castellano, J.; Reina, E. Comet 22P/Kopff: Dust environment and grain ejection anisotropy from visible and infrared observations. Astrophys. J. 2011, 752, 136, doi:10.1088/0004-637X/752/2/136. [CrossRef]

50. Capistrano, A.J.S.; Barrocas, G.R.G. Rotation curves of LSBGs and dwarf galaxies in a nearly-Newtonian solution. Mon. Not. R. Astron. Soc. 2018, 475, 2204-2214, doi:10.1093/mnras/stx2909. [CrossRef]

51. Rubin, V.C.; Kent Ford, W., Jr. Rotation of the Andromeda Nebula from a Spectroscopic Survey of Emission Regions. Astrophys. J. 1970, 159, 379-403, doi:10.1086/150317. [CrossRef]

52. Sanders, R.H. The Dark Matter Problem-A Historical Perspective; Cambridge University Press: New York, NY, USA, 2010; ISBN 9780521113014.

53. Bertone, B; Hooper, D. A History of dark matter. arXiv 2016, arXiv:1605.04909.

54. Lelli, F.; McGaugh, S.S.; Schombert, J.M.; Pawlowski, M.S. One Law to Rule Them All: The Radial Acceleration Relation of Galaxies. Astrophys. J. 2017, 836, 152, doi:10.3847/1538-4357/836/2/152. [CrossRef]

55. Blumenthal, G.R.; Faber, S.M.; Flores, V.; Primack, J.R. Contraction of dark matter galactic halos due to baryonic infall. Astrophys. J. 1984, 301, 27-34, doi:10.1086/163867. [CrossRef]

56. de Blok, W.J.G.; McGaugh, S.S.; Rubin, V.C. High-Resolution Rotation Curves of Low Surface Brightness Galaxies. I. Data. Astrophys. J. 2001, 122, 5, doi:10.1086/323448. [CrossRef]

57. Karukes, E.V.; Salucci, P. The universal rotation curve of dwarf disc galaxies. Mon. Not. R. Astron. Soc. 2017, 465, 4703-4722, doi:10.1093/mnras/stw3055. [CrossRef]

58. Hartle, J.B. Gravity: An Introduction To Einstein's General Relativity; Addison-Wesley Professional: San Francisco, CA, USA, 2003; pp. 126-130; ISBN 080-538-662-9.

59. Griffiths, J.B.; Podolsky, J. Exact Space-Times in Einstein's General Relativity; Cambridge University Press: Cambridge, UK, 2009; Chapter 10; ISBN 110-740-618-8.

60. Cooperstock, F.I.; Tieu, S. General Relativity resolves galactic rotation without exotic Dark Matter. arXiv 2005, arXiv:astro-ph/0507619.

(C) 2018 by the author. Licensee MDPI, Basel, Switzerland. This article is an open access article distributed under the terms and conditions of the Creative Commons Attribution (CC BY) license (http://creativecommons.org/licenses/by/4.0/). 